\title{
Assessment of Lung Parenchyma Recovery after Antibiotic Administration using Lung Ultrasound in Critically III Patients with Pneumonia
}

\author{
MaiAdelSahbal*(iD, Mohammed OmarAlghoneimy (i), SallySalahEldine (iD,AmrElsayed Elhadidy (D), Mahmoud Muhammad Kenawy (iD \\ Department of Critical Care Medicine, Cairo University, Giza, Egypt
}

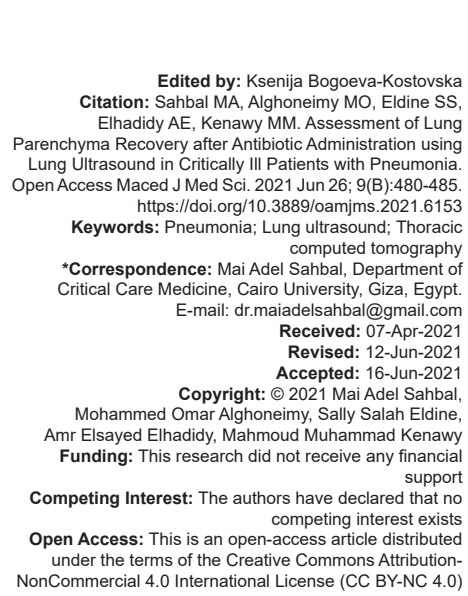

\section{Introduction}

Pneumonia is a common infection among intensive care unit (ICU) patients either as a primary cause or a complication following hospital admission [1]. Pneumonia is defined as an inflammation of lung parenchyma, in which the affected part is consolidated and the alveoli are filled with inflammatory exudate. Infection by bacteria or viruses is the most common cause [2].

Pneumonia is divided into community acquired, hospital acquired, and ventilator associated (CAP/HAP and VAP) according to the American Thoracic Society (ATS) guidelines [3], [4]. Diagnosis and follow-up require clinical assessment, radiographical examination, and microbiological tests [5].

Radiological assessment by bedside chest radiography (CXR) showed poor accuracy and multiple limitations [6], [7] that made thoracic computed tomography (CT) the gold standard. Nowadays, bedside lung ultrasound (LUS) is increasingly used; being noninvasive, easily repeatable, with less radiation exposure and no risk of transfer compared to CT [6]. Our study aimed to detect the accuracy of LUS in predicting parenchymal lung recovery following antibiotic administration in critically ill patients with pneumonia.

\section{Methods}

\section{The study population}

Fifty patients were examined when presented to Critical Care Department, Cairo University, with pneumonia according to the ATS definitions [3], [4]. 


\section{Inclusion criteria}

1. CAP patients admitted to the ICU either due to CURB-65 $>2$ or other indication for ICU admission

2. HAP patients needed ICU admission due to either severe sepsis, hemodynamic instability, and need for ventilatory support or secondary to another indication apart from pneumonia 3. VAP patients.

\section{Exclusion criteria}

Patients $<18$ years of age, referred from another ICU and those who were suffering from acquired immunodeficiency syndrome-defining condition, or neutropenia $\left(P N L s<1000 / \mathrm{mm}^{3}\right.$ ), were excluded from the study.

\section{Test methods}

\section{Study design}

This was a cross-sectional study.

\section{Data collection and study setting}

This study was conducted from October 2018 to October 2019 at the Critical Care Department, Cairo University, Cairo, Egypt.

All patients were subjected to demographic data collection, medical history, thorough clinical examination, ventilator support settings - if needed and blood, sputum/endotracheal cultures. Patients were managed with antimicrobial therapy protocol according to the ATS guidelines.

Scoring systems were conducted for all patients as follows; (I) acute physiology and chronic health evaluation II score was calculated for all patients at day 0 and analyzed for final outcome. (II) CURB65 score in patients with CAP (III) modified clinical pulmonary infection score (CPIS) score in HAP/VAP at day of diagnosis (day 0 ) then both were repeated on day 3,5 , and 7 and analyzed for antibiotic response. Final outcome was assessed at day 28 for death or survival.

\section{LUS protocol}

LUS was performed at days $0,3,5$, and 7 using ultrasound (US) machine manufactured by Siemens electronics (Sonoline Adara) with a regular size convex probe (5 $\mathrm{MH}$ frequency).

With patient lying in the supine position, each hemithorax is divided into six areas; 1 and 2 refer to superior and basal anterior areas, respectively; extending between the parasternal line and the anterior axillary line, 3 and 4 refer to superior and basal lateral areas extending between anterior and posterior axillary line; and 5 and 6 refer to superior and basal posterior areas extending between the posterior axillary and the paravertebral lines [8]. Intercostal spaces of upper and lower parts of the different regions of both lungs were examined, and videos were saved.

The transducer was held perpendicular to the skin surface with the transducer marker (groove) pointed cephalic and the scanning plane directed between adjacent ribs, each region was examined. All lung areas were examined at day 0 and described according to the presence and shape of $B$ lines and consolidation as follows:

B1 for non-coalescent B lines with $7 \mathrm{~mm}$ apart; B2 for coalescent B lines; C for lung consolidation; and $\mathrm{N}$ for normal pattern (Table 1)[9]. At day 3, lung areas were examined and each lung area was given a score according to shift from previous pattern and then all lung areas scores were summed giving a final score. At day 5 , findings were compared to those of day 3 , US score was calculated then delta score is also calculated to detect best cutoff value for response and reaeration. Finally, lung areas were examined at day 7 and lung score was calculated the same as day 5.

Table 1: LUS score for quantification of loss of aeration and reaeration

\begin{tabular}{|c|c|c|c|c|c|}
\hline \multicolumn{3}{|c|}{ Quantification of reaeration } & \multicolumn{3}{|c|}{ Quantification of loss of aeration } \\
\hline 1 point & 3 points & 5 points & -5 points & -3 points & -1 point \\
\hline $\mathrm{B} 1 \Rightarrow \mathrm{N}$ & $B 2 \Rightarrow N$ & $\mathrm{C} \Leftrightarrow \mathrm{N}$ & $\mathrm{N} \Leftrightarrow \mathrm{C}$ & $\mathrm{N} \Leftrightarrow \mathrm{B} 2$ & $\mathrm{~N} \Rightarrow \mathrm{B} 1$ \\
\hline $\mathrm{B} 2 \Rightarrow \mathrm{B} 1$ & $\mathrm{C} \Leftrightarrow \mathrm{B} 1$ & - & - & $B 1 \Rightarrow C$ & $\mathrm{~B} 1 \Rightarrow \mathrm{B} 2$ \\
\hline $\mathrm{C} \Leftrightarrow \mathrm{B} 2$ & - & - & - & - & $B 2 \Rightarrow C$ \\
\hline
\end{tabular}

Air bronchogram is detected whenever seen, it refers to the phenomenon of air-filled bronchi that became visible by the opacification of surrounding alveoli. With lung hepatization, dynamic air bronchograms make pneumonia more likely, while static or no bronchograms make atelectasis more likely.

\section{Culture technique}

Sputum culture was obtained from nonventilated patients and endotracheal aspirate (ETA) or mini-bronchoalveolar lavage (BAL) was performed in ventilated patients at day 0 and repeated at days 3,5 , and 7 as a part of modified CPIS score. Laboratory was informed in case of previous antibiotics use. Samples were processed within $2 \mathrm{~h}$ if kept at room temperature and within $24 \mathrm{~h}$ if kept at $4^{\circ} \mathrm{C}$. Cultures were considered positive once thresholds to diagnose pneumonia were detected: a PSB of $>10^{3} \mathrm{CFU} / \mathrm{mL}$, a mini-BAL of $>10^{4} \mathrm{CFU} / \mathrm{mL}$, and ETA $>10^{5} \mathrm{CFU} / \mathrm{mL}$ [10].

\section{CT protocol}

Lung scanning without administering intravenous contrast was performed from the apex to the diaphragm with a $15 \mathrm{~s}$ breath hold in spontaneously 
breathing patients and induced apnea in ventilated patients. Adjacent axial CT sections $10 \mathrm{~mm}$ thick were reconstructed from the volumetric data [11] and recorded. On each $\mathrm{CT}$ section, lung parenchyma was manually delineated [12]. The total volume of gas was computed as $(-\mathrm{CT} / 1000)$ total volume (total number of voxels), where CT is the CT attenuation of voxels with a CT number 0 using the software Lung view [13].

Parenchymal lung recovery and lung reaeration resulting from antibiotics administration were defined as the additional volume of gas detected within both lungs following 5 days of antimicrobial therapy protocol.

To provide similar conditions of measurements at day 0 and day 7 , LUS and CT were consecutively performed in the supine position at zero end-expiratory pressure. Each exam was separated by $2 \mathrm{~h}$ time interval to regain positive end-expiratory pressure and avoid atelectatic complications in mechanically ventilated patients.

Using this time scheduled follow-up of clinical and microbiological variables; pneumonia was considered as successfully treated by antimicrobial therapy if any of the following criteria were met: (1) Decrease in CPIS for VAP patients; (2) CT reaeration corresponding to partial or complete regression of consolidations and rounded CT attenuations; and (3) either extubation between day 4 and 7 or negative mini-BAL in patients remaining ventilated at day 7 .

\section{Statistical methods}

Data were coded and entered using the Statistical Package for the Social Sciences version 25. Data were summarized using mean, standard deviation, median, minimum and maximum in quantitative data, and using frequency (count) and relative frequency (percentage) for categorical data. Comparisons between quantitative variables were done using the non-parametric MannWhitney U-test. For comparing categorical data, Chi-square $\left(\chi^{2}\right)$ test was performed. Exact test was used instead when the expected frequency is $<5$. Standard diagnostic indices including sensitivity, specificity, positive predictive value (PPV), negative predictive value (NPV), and diagnostic efficacy were calculated. Receiver operating characteristic (ROC) curve was constructed with area under curve analysis performed to detect best cutoff value of LUS at day 5 and 7 for detection of improvement. $p<0.05$ was considered as statistically significant. Kappa measure of agreement was used to test agreement between categorical variables.

\section{Results}

\section{Participants}

Fifty-seven patients were eligible for the study, 50 patients were finally enrolled and analyzed as shown in patients flowchart (Figure 1), Twenty-one males $(42 \%)$ and twenty-nine (58\%) females were enrolled in the study, with mean age of $63 \pm 18$ years. About $40 \%$ of patients were smokers, $50 \%$ were hypertensive, and $52 \%$ were diabetic.

The studied population included 10 patients $(20 \%)$ with CAP, 16 (32\%) with early HAP, and 24 patients (48\%) with VAP. Best pneumonia response and final outcome were detected among CAP patients with $100 \%$ success of treatment and 28 days survival, while patients with HAP and VAP showed $37.5 \%$ mortality. Six cases of HAP patients died, four of them with worsening pneumonia, one case due to surgical complications, and the second case secondary to $\mathrm{Ml}$ and cardiogenic shock. VAP cases showed worsening and death of nine cases of the 15 studied patients.

Highest mortality was seen among patients infected with mixed Gram-negative bacteria (66\%) followed by patients with Acinetobacter infection (50\%) then those with Klebsiella (37.5\%).

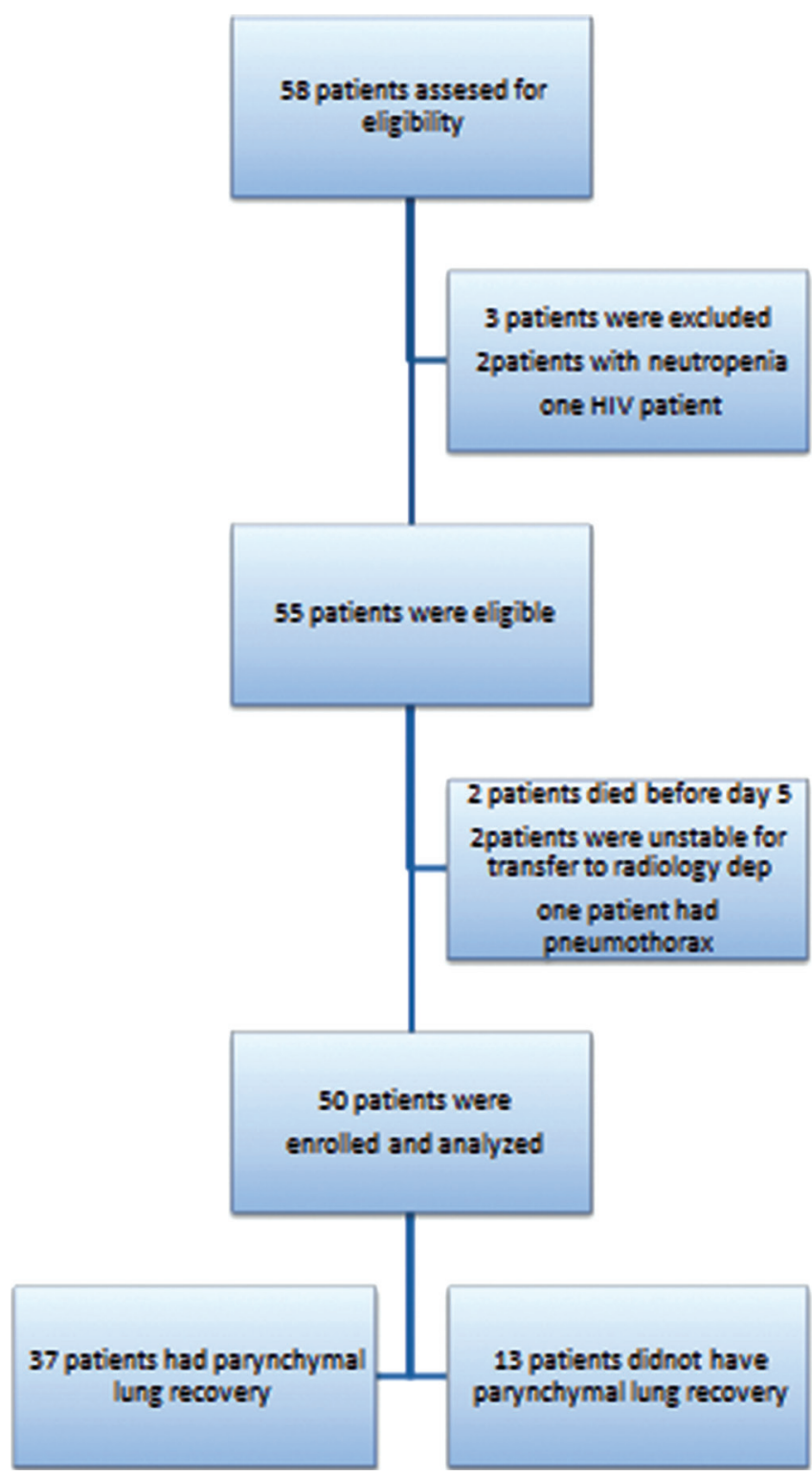

Figure 1: Flowchart of patients included in the study 


\section{Test results and estimates of diagnostic accuracy}

In 37 patients, pneumonia was considered as successfully treated by antimicrobial therapy on the following arguments: (a) The mean CPIS for HAP and VAP patients had decreased to 4.3 at day 7 , CURB 65 score for all CAP patients decreased at day 7 to mean of 1.5 ; (b) a significant CT reaeration was observed at day 7 together with a partial regression or a complete disappearance of consolidations and round CT attenuations that were present at day 0; (c) 15 patients were extubated between day 4 and 7; and (d) mini-BALs performed in the 15 patients who remained ventilated were negative at day 7 .

In 13 patients, VAP and HAP were considered as unsuccessfully treated by antimicrobial therapy on the following arguments: (a) The mean CPIS remained at 6.8; (b) in eight patients, no significant $\mathrm{CT}$ reaeration was observed at day 7, corresponding to persisting consolidations and round CT attenuations present at day 1 ; (c) in five patients, no significant $\mathrm{CT}$ reaeration was observed at day 7 , corresponding to new consolidations and round CT attenuations, whereas consolidations and round $\mathrm{CT}$ attenuations present at day 0 had partially disappeared; (d) the 13 patients were still on mechanical ventilation at day 7 ; and (e) mini-BALs performed in the 13 patients were all positive at day 7 .

Therapeutic failure was related to lack of eradication of causative microorganism in four patients and to secondary lung infection by another microorganism resistive to the antimicrobial therapy in four patients. Lung morphology characterizing ventilator-associated pneumonia, in nine patients, VAP was exclusively characterized by intraparenchymal and subpleural rounded CT attenuations disseminated within upper and/or lower lobes. In five patients, VAP was exclusively characterized by consolidations of lower lobes associated or not with consolidation of upper lobes. In 36 patients, VAP was characterized by an association of consolidations affecting one or several lobes with intraparenchymal and subpleural rounded CT attenuations disseminated within upper and/or lower lobes. Rounded CT attenuations were either isolated or confluent, forming more or less extended ground glass areas. Their size ranged between 1 and $15 \mathrm{~mm}$, and many of them were subpleural, surrounded by normally aerated lung parenchyma.

Comparing CT chest and LUS showed improvement of 34 patients and worsening of 12 patients in both. Disagreement was detected between the two tests in four cases, one case showed improvement in lung US score but not in CT chest and the other three cases showd improvement in CT chest only.

Lung US score ranged from -2 to 17 with mean value of $8.75 \pm 3.88$ for improving patients. patients, while US score in patients with worsening pneumonia ranged from -20 to 2 with mean value of $-10.08 \pm 6.95$ with high statistical significance (Table 2).
Table 2: LUS score at day 7 between the two groups

\begin{tabular}{|c|c|c|c|c|c|c|c|}
\hline \multirow[t]{3}{*}{ US score } & \multicolumn{6}{|c|}{ Pneumonia response } & \multirow[t]{3}{*}{$\mathrm{p}$-value } \\
\hline & \multicolumn{3}{|l|}{ Improved } & \multicolumn{3}{|l|}{ Worsened } & \\
\hline & $\overline{M e a n} \pm$ SD & Min & Max & $\overline{M e a n} \pm$ SD & Min & $\overline{M a x}$ & \\
\hline LUS score at day 7 & $8.57 \pm 3.88$ & -2.00 & 17.00 & $-10.08 \pm 6.95$ & -20.00 & 2.00 & $<0.001^{*}$ \\
\hline
\end{tabular}
LUS: Lund ultrasound

US score at day 7 showed excellent sensitivity and specificity of $91.89 \%$ and $92.31 \%$, respectively, when compared to CT, with PPV of $97.14 \%$ and NPV of $80 \%$ and accuracy of $92 \%$ with strong statistical significance $(p<0.001)$.

Best cutoff value of LUS score for detecting good response to antibiotics was 2.5 ( $p<0.001$ ), with lower bound of 0.975 , upper bound of 1.000 , and area under the curve (AUC) of 0.992 and with $91.9 \%$ sensitivity and $100 \%$ specificity, with $p$ value $>0.001$ and confidence interval $(\mathrm{Cl})$ of $95 \%$ (Table 4 ).

US score showed high agreement with CT when running Kappa statistics between the two judgments (Table 3).

Table 3: Agreement between US score, air bronchogram, $B$ lines, and CT at day 7

\begin{tabular}{llllll}
\hline US variables & & Value & $\begin{array}{l}\text { Asymptomatic } \\
\text { standard error }\end{array}$ & Approximate T & p-value \\
\hline US score & Kappa & 0.802 & 0.094 & 5.699 & $<0.001^{*}$ \\
Air bronchogram & Kappa & 0.520 & 0.139 & 3.684 & 0.001 \\
B lines & Kappa & 0.307 & 0.131 & 2.312 & 0.027 \\
\hline US: Ultrasound, CT: Computed tomography. & & &
\end{tabular}

Sub-analysis of air bronchogram showed that $27(54 \%)$ patients had a dynamic air bronchogram and $16(32 \%)$ patients had a static air bronchogram. After treatment, in $24(48 \%)$ patients, the air bronchogram disappeared and resolved (totally reaerated); in $6(12 \%)$ patients. Air bronchogram became dynamic after being static (partially reaerated); and in 7 (14\%) patients, the air bronchogram was static: In the latter case, in $1(2 \%)$ case, the dynamic air bronchogram became static, and in $6(12 \%)$ patients, the static air bronchogram remained static with $p>0.001$, sensitivity of $61.5 \%$ and specificity of $89.1 \%$ and with PPV of $66.67 \%$ and NPV of $86.84 \%$ and accuracy of $82 \%$ and with moderate agreement between the air bronchogram and CT when running Kappa statistics between two judgments (Table 4).

Table 4: B-lines and air bronchogram progression between day 0 and day 7

\begin{tabular}{|c|c|c|c|c|c|}
\hline \multirow{2}{*}{$\begin{array}{l}\text { US variables } \\
\text { analysis }\end{array}$} & \multicolumn{2}{|l|}{ Day 0} & \multicolumn{2}{|l|}{ Day 7} & \multirow[t]{2}{*}{$\mathrm{p}$-value } \\
\hline & Count & $\%$ & Count & $\%$ & \\
\hline \multicolumn{6}{|l|}{ Air bronchogram } \\
\hline Resolved & 7 & 14.0 & 33 & 66.0 & \multirow[t]{3}{*}{$<0.001$} \\
\hline Dynamic & 27 & 54.0 & 10 & 20.0 & \\
\hline Static & 16 & 32.0 & 7 & 14.0 & \\
\hline \multicolumn{6}{|l|}{ B-lines } \\
\hline Present & 50 & 100.0 & 12 & 24.0 & \multirow[t]{2}{*}{$<0.001$} \\
\hline absent/improved & 0 & 0.0 & 38 & 76.0 & \\
\hline
\end{tabular}

Studying B-lines before antibiotic administration (day 0) showed that $100 \%$ of patients had B-lines versus $24 \%$ at day 7 after antibiotic administration with $p<0.001$ with sensitivity of $69.2 \%$ and specificity of $67.5 \%$ and accuracy of $68 \%$ but with fair (0.31) agreement with CT chest $p<0.027$ (Table 3). 


\section{Discussion}

Pneumonia is a common infection worldwide with increasing use of LUS among patients; especially those in ICUs. In our study, lung US score at day 7 showed excellent sensitivity and specificity when compared to pneumonia response (CT reaeration at day 7) of $91.89 \%$ and $92.31 \%$, respectively, with PPV of $97.14 \%$ and NPV of $80 \%$ and accuracy of $92 \%$.

Similarly, Peris et al. reported the effectiveness of bedside US in the ICU setting with decline in the use of chest radiographs (26\%) and CT scans (47\%) [14]. Furthermore, Vitturi et al. reported good concordance between chest US when compared with chest CT in diagnosing different lung diseases [15].

Another study performed by Lichtenstein and Mezière compared bedside CXR diagnostic accuracy with that of LUS to report much higher accuracy for LUS in diagnosing pleural effusion, alveolar consolidation, and alveolar interstitial syndrome [16].

In our study, we detected that change of LUS score by 2.5 as a best cutoff value $(p<0.001)$ reflecting good antibiotic response with a sensitivity of 91.9 and specificity of $100 \%$. When El-Moursi et al. studied accuracy of US score in the assessment of pulmonary reaeration in VAP after antibiotic administration, the ROC curve for chest US reported 1.000 as best cutoff value for US assessment score $(p<0.001)$ with sensitivity of $100 \%$ and specificity of $100 \%$ [17].

They reported that $100 \%$ of patients had B-lines at day 0 versus $24 \%$ at day 5 after antibiotic administration with $p<0.001$, which means that the B-lines were significant for assessing lung reaeration and good antibiotic response. Furthermore, Cortellaro et al. and Lichtenstein have demonstrated that patients with VAP [16], [18] and with ground-glass opacification on CT would have B-lines in lung US and that B-line correlates with interstitial edema may be focal or diffuse. Also Bouhemad et al. described antibiotic response by lung US in VAP patients with disappearance of B-lines and comet tails or transformation of lung consolidation into comet tails [8].

Inversely, poor antibiotic response was associated with new appearance of lung comets $(87 \%)$, consolidation $(3 \%)$, or transformation comets into consolidation $(7 \%)$ in LUS, With corresponding CT changes in the form of appearance of new rounded opacities disseminated within both lungs [19].

Bouhemad et al. reported poor accuracy of CXR in detecting changes in lung aeration after antimicrobial therapy in contrast to LUS that could detect antibiotic effectiveness in the follow-up of VAP when compared with CT scan. Whereas antibiotic failure corresponds to new subpleural consolidation appearance or coalescing in lobar consolidation. In this study on VAP patients, lung reaeration after 7 days of antimicrobial therapy was measured by CT scan and compared with LUS reaeration score [8].

\section{Conclusion}

The chest US is reasonable tool for assessing parenchymal lung recovery in patients with pneumonia who are successfully treated with antibiotics with very good sensitivity and specificity in comparison with CT chest. It is reasonable bedside method, especially for unstable patients for whom transfer for CT chest carries considerable risk.

\section{Future Research Implications}

Conducting similar studies with larger number of patients to confirm results and study lung US ability to detect pneumonia complications and presence of underlying pathology in comparison with CT.

\section{Study Limitations}

All the ICU research teams were not gathered except for the time of their scheduled shifts and rounds, so patient examination was delivered individually not in groups.

\section{Acknowledgment}

The research team especially thanks each and everyone in the critical care department at Cairo University Hospital for their utmost help and welcome that was priceless for completion of this research.

\section{References}

1. Lichtenstein DA. Ultrasound in the management of thoracic disease. Crit Care Med. 2007;35(Suppl):S250-61.

2. Reissig A, Kroegel C. Sonographic diagnosis and follow-up of pneumonia: A prospective study. Respiration. 2007;74(5):53747. https://doi.org/10.1159/000100427 PMid:17337882

3. Govindan S, Hyzy RC (2016) The 2016 guidelines for hospitalacquired and ventilator-associated pneumonia. A selection correction? Am J Respir Crit Care Med 194:658-660. https:// doi.org/10.1164/rccm.201607-1447ED 
4. Mandell LA, Wunderink RG, Anzueto A, Bartlett JG, Campbell GD, Dean NC, Dowell SF, File TM Jr, Musher DM, Niederman MS, Torres A, Whitney CG; Infectious Diseases Society of America; American Thoracic Society. Infectious Diseases Society of America/American Thoracic Society consensus guidelines on the management of community-acquired pneumonia in adults. Clin Infect Dis. 2007 Mar 1;44 Suppl 2(Suppl 2):S27-72. doi: 10.1086/511159. PMID: 17278083;

PMCID: PMC7107997.

5. Zilberberg MD, Shorr AF. Ventilator-associated pneumonia: the clinical pulmonary infection score as a surrogate for diagnostics and outcome. Clin Infect Dis. 2010;51(Suppl 1):S131-5. https:// doi.org/10.1086/653062

PMid:20597663

6. Rubinowitz AN, Siegel MD, Tocino I. Thoracic imaging in the ICU. Crit Care Clin. 2007;23(3):539-73. https://doi.org/10.1016/j. ccc.2007.06.001

PMid: 17900484

7. Henschke $\mathrm{Cl}$, Yankelevitz DF, Wand A, Davis SD, Shiau M. Accuracy and efficacy of chest radiography in the intensive care unit. Radiol Clin North Am. 1996;34(1):21-31.

PMid:8539351

8. Bouhemad B, Zhang M, Lu Q, Rouby JJ. Clinical review: Bedside lung ultrasound in critical care practice. Crit Care. 2007;11(1):205. https://doi.org/10.1186/cc5668 PMid: 17316468

9. Bouhemad B, Liu ZH, Arbelot C, Zhang M, Ferarri F, Le-Guen $M$, et al. Ultrasound assessment of antibiotic-induced pulmonary reaeration in ventilator-associated pneumonia. Crit Care Med. 2009;38(1):84-92. https://doi.org/10.1097/ ccm.0b013e3181b08cdb PMid:19633538

10. Raman K, Nailor MD, Nicolau DP, Aslanzadeh J, Nadeau M, Kuti JL. Early antibiotic discontinuation in patients with clinically suspected ventilator-associated pneumonia and negative quantitative bronchoscopy cultures. Crit Care Med. 2013;41(7):1656-63. https://doi.org/10.1097/ ccm.0b013e318287f713 PMid:23528805

11. Lu Q, Malbouisson M, Mourgeon E, Goldstein I, Coriat P, Rouby JJ. Assessment of PEEP-induced reopening of collapsed lung regions in acute lung injury: Are one or three CT sections representative of the entire lung? Intensive Care Med. 2001;27(9):1504-10. https://doi.org/10.1007/s001340101049

PMid:11685344

12. Puybasset L, Cluzel P, Gusman P, Grenier P, Preteux F, Rouby JJ. Regional distribution of gas and tissue in acute respiratory distress syndrome, I: Consequences for lung morphology. CT Scan ARDS Study Group. Intensive Care Med. 2000;26(7):857-69. https://doi.org/10.1007/s001340051274 PMid:10990099

13. Malbouisson LM, Preteux F, Puybasset L, Grenier $P$, Coriat $P$, Rouby JJ. Validation of a software designed for computed tomographic (CT) measurement of lung water. Intensive Care Med. 2001;27:602-8. https://doi.org/10.1007/s001340100860

14. Peris A, Tutino L, Zagli G, Batacchi S, Cianchi G, Spina R, et al. The use of point-of-care bedside lung ultrasound significantly reduces the number of radiographs and computed tomography scans in critically ill patients. Anesth Analg. 2010;111(3):687-92. https://doi.org/10.1213/ane.0b013e3181e7cc42 PMid:20733164

15. Vitturi N, Dugo M, Soattin M, Simoni F, Maresca L, Zagatti R, et al. Lung ultrasound during hemodialysis: The role in the assessment of volume status. Int Urol Nephrol. 2014;46(1):16974. https://doi.org/10.1007/s11255-013-0500-5 PMid:23884727

16. Lichtenstein DA, Mezière GA. Relevance of lung ultrasound in the diagnosis of acute respiratory failure: The BLUE protocol. Chest. 2008;134(1):117-25. https://doi.org/10.1378/ chest.07-2800

PMid:18403664

17. El-Moursia AA, Besheya BN. Ultrasound assessment of antibiotic-induced pulmonary reaeration in ventilator-associated pneumonia. Res Opin Anesth Intens Care. 2017;4:7-16. https:// doi.org/10.4103/2356-9115.202699

18. Cortellaro F, Colombo S, Coen D, Duca PG. Lung ultrasound is an accurate diagnostic tool for the diagnosis of pneumonia in the emergency department. Emerg Med J. 2012;29(1):19-23. https://doi.org/10.1136/emj.2010.101584 PMid:21030550

19. Beckh S, Bölcskei PL, Lessnau KD. Real-time chest ultrasonography: A comprehensive review for the pulmonologist. Chest. 2002;122(5):1759-73. https://doi.org/10.1378/ chest.122.5.1759

PMid:12426282 